\title{
Fiskalpolitik in der Corona-Krise: Wie viel ist zu viel?
}

Die wirtschaftliche Erholung von der Corona-Pandemie verläuft global sehr unterschiedlich. Während Chinas Wirtschaft 2020 als einzige große Volkswirtschaft mit 2,3\% wuchs und sich 2021 mit über $8 \%$ Wachstum weiter schnell erholen wird, befinden sich auch die USA - wesentlich gestützt durch eine erfolgreiche Impfkampagne - auf Erholungskurs. Der Einbruch 2020 war mit einem Rückgang von 3,5\% vergleichsweise moderat, die Erholung wird 2021 mit gut $6 \%$ unerwartet dynamisch verlaufen (vgl. Abbildung 1). Die Eurozone hingegen verzeichnete mit rund minus $7 \%$ einen deutlich stärkeren Einbruch und mit einem Plus von gut $4 \% 2021$ eine deutlich schwächere Erholung. Auch in Japan und - vor allem bedingt durch den Brexit in Großbritannien wird die konjunkturelle Dynamik schwächer sein. Die unterschiedlichen Erholungsdynamiken können sich in den nächsten Jahren als eine Belastung für die Weltwirtschaft und insbesondere für die internationale Koordination der Geld- und Fiskalpolitik erweisen, das gilt insbesondere für die Eurozone, für die pandemiebedingt eine zunehmende strukturelle Divergenz zu erwarten ist, was sich zu einem strukturellen Problem verfestigen kann.

Neben der epidemiologischen Bekämpfung der Pandemie ist vor allem der fiskalpolitische Spielraum ein wichtiger Faktor für die mittelfristige Erholung von der Krise. Die fiskalpolitische Antwort auf die Pandemie fällt sehr unterschiedlich aus. In Japan, den USA und Großbritannien fiel sie im Jahr 2020 mit über $5 \%$ des Bruttoinlandsprodukts (BIP) am größten aus (vgl. Abbildung 2). Die neue USRegierung von Präsident Joe Biden hat im März 2021 ein weiteres Fiskalpaket über rund 1,9 Billionen US-\$ verabschiedet; das sind noch einmal rund $10 \%$ des BIP zusätzlich zu dem bisherigen Impuls. Darüber ist in den USA eine Debatte entbrannt, ob es nicht nur ein Zuwenig, sondern auch ein Zuviel in der fiskal- und konjunkturpolitischen Überwindung der Pandemie geben kann.

Die Corona-Pandemie ist der größte globale Schock für die Weltwirtschaft seit vielen Jahrzehnten. Die spezielle Natur einer Pandemie erzeugt zugleich eine spezielle Ökonomik des Schocks, der aus einer Mischung aus Angebots- und Nachfrageschock besteht. Außerdem erzeugt eine Pande-

(c) Der/die Autor:in(nen) 2021. Open Access: Dieser Artikel wird unter der Creative Commons Namensnennung 4.0 International Lizenz veröffentlicht (creativecommons.org/licenses/by/4.0/deed.de).

Open Access wird durch die ZBW - Leibniz-Informationszentrum Wirtschaft gefördert. mie eine hohe Unsicherheit über den künftigen Verlauf und dadurch eine hohe Abhängigkeit der wirtschaftlichen Aktivität vom Pandemiegeschehen. Die epidemiologischen Maßnahmen zur Eindämmung interagieren folglich aufs Engste mit den wirtschafts- und konjunkturpolitischen Instrumenten zur Abfederung der wirtschaftlichen Folgen. Es stellt sich die Frage, welche Instrumente in diesem Fall wirksam und effizient sind, wie also der fiskalpolitische Impuls ausgestaltet sein sollte, um das aggregierte Angebot zu schützen und die aggregierte Nachfrage zu stützen. Insbesondere die dynamische Wirkung und die intertemporalen Trade-offs der fiskalpolitischen Maßnahmen sind entscheidend, sowohl in Bezug auf die kurzfristige als auch auf die mittelfristigen Wirkungen der Fiskalpolitik. Die Pandemie stellt angesichts der extrem hohen wirtschaftlichen Kosten einen „Whatever it takes"-Moment der Fiskalpolitik dar. Mit dem Comeback der Fiskalpolitik verbinden einige Ökonomen und Politiker sogar ein neues makroökonomisches Paradigma der Wirtschaftspolitik. Weit weniger klar ist jedoch, welche Rolle die Fiskalpolitik im Ausgang der Pandemie spielen sollte. Kann die Wirtschaft mehr oder weniger von allein hochfahren oder benötigt sie den Anschub durch öffentliche Ausgaben? Wie groß ist dabei der Effekt der reinen Nachfragesteuerung und wie stark angebotsorientiert und transformativ sollten öffentliche Investitionen angesichts des parallelen Strukturwandels sein? Wie viel wäre zu viel des Guten im Hinblick auf die mittel- bis langfristigen Folgen der Fiskalpolitik?

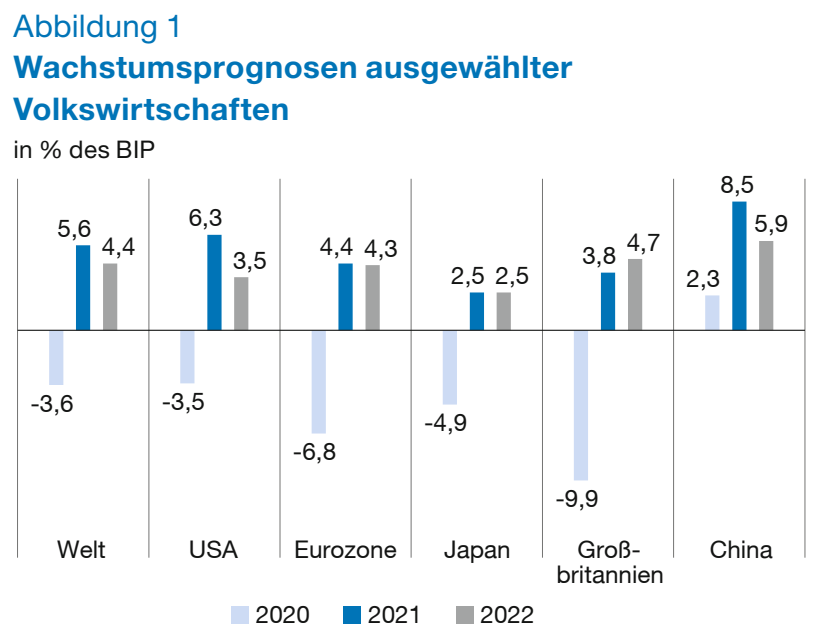

Quelle: Consensus Forecast, Peterson Institute for International Economics (2021). 
Abbildung 2

Fiskalimpulse ausgewählter Volkswirtschaften im Jahr 20201

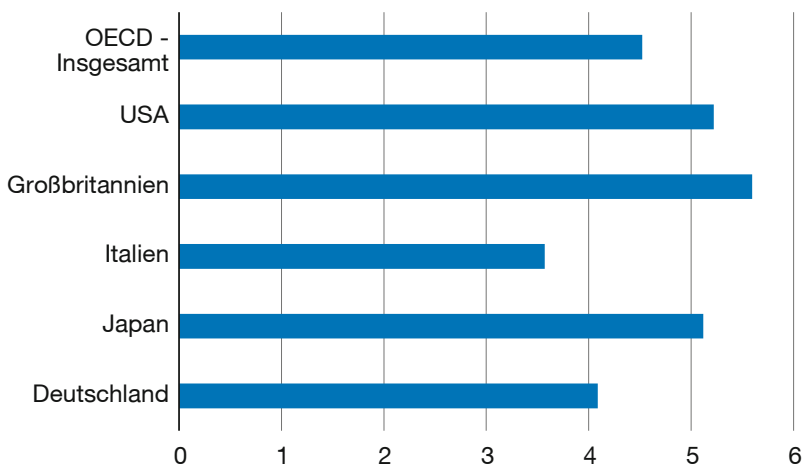

Anmerkungen: ${ }^{1}$ Veränderung des konjunkturbereinigten gesamtstaatlichen Finanzierungsdefizits 2020 gegenüber 2019 in \% des Bruttoinlandsprodukts.

Quelle: OECD (2020).

Larry Summers (2021) etwa hält den abermaligen fiskalischen Stimulus der Biden-Regierung für überzogen; er sei größer als die Nachfragelücke und könne über eine konjunkturelle Überhitzung mittelfristig Inflation auslösen. Summers sprach in einem Interview mit Bloomberg weiter von der unverantwortlichsten Fiskalpolitik der letzten 40 Jahre. Einem seiner Tweets zufolge gäbe es jeweils eine Drittel-Wahrscheinlichkeit für die Szenarien einer Stagflation, einer Rezession und eines inflationsfreien Wachstums. Brad DeLong (2021) sieht dagegen in der Pandemie keinen Angebots-Kosten-Schock, der typischerweise für eine Stagflation im Stil der 1970er Jahre ursächlich sein könnte. Entsprechend würde der Fiskalimpuls eher einen inflationären Nachfrage-Boom auslösen. Befürworter eines stärkeren fiskalischen Impulses, darunter J. W. Mason (2021) und Paul Krugman (2021), argumentieren, dass die Output-Lücke und die Arbeitslosigkeit am aktuellen Rand unterschätzt würden. Durch die Pandemie seien derzeit noch rund 8 Mio. Arbeitsplätze im Vorkrisenvergleich verlorengegangen, rund 2 Mio. wären in dieser Zeit zusätzlich entstanden. Der fiskalische Impuls kann und sollte entsprechend expansiv ausfallen, zumal die makroökonomischen Kosten und Risiken asymmetrisch verteilt seien. Durch rigide Löhne sind Abweichungen von der Zielinflationsrate nach unten teurer als Abweichungen nach oben. Mason führt in seiner Argumentation für den American Rescue Plan Act (ARPA) weiterhin aus, dass Hysterese-Effekte nach Krisen das Produktivitätswachstum hemmen würden. Alles in allem sei also ein Zuviel an fiskalischem Impuls unwahrscheinlich bis nahezu ausgeschlossen. Die Gefahr, dass die Corona-Pandemie ohne einen hinreichend großen Stimulus zu säkularer Stagnation, ein ironischerweise durch Larry Summers (2015) vor Jahren in die Debatte zurückgekehrter und ursprünglich von Alvin Hansen eingeführter Begriff, führen könne, sei groß. Gerade unter den Bedingungen mittelfristig niedriger Zinsen und relativer Ineffektivität der Geldpolitik wird eine größere stabilitätspolitische Bedeutung der Fiskalpolitik zur Herstellung von Vollbeschäftigung gesehen. Der ehemalige Präsident der Bank of England, Mervyn King (2021) hingegen fragt, weshalb eine gesamtwirtschaftliche Nachfrage stimuliert werden solle, die ohnehin von allein wiederkomme.

Die US-Fiskalpolitik unter Biden darf aber nicht allein unter konjunkturpolitischen Aspekten betrachtet werden. Sie verfolgt erkennbar eine größere wirtschaftspolitische Agenda, die bereits unter dem Stichwort "Bidenomics" diskutiert wird. Sein Versprechen, das er in seiner Inauguration gab, nämlich die US-amerikanische Gesellschaft und Nation zu heilen, erfordert eine inklusivere und nachhaltigere Wirtschaftspolitik. Gleichwohl stellen sich einige Fragen hinsichtlich der mittelfristigen Folgen eines solchen Schwenks in der Fiskalpolitik, denn in Krisen hieß es immer: „This time is different." Neben den möglichen inflationären Folgen durch eine Überhitzung stellt sich vor allem die Frage nach der Schuldentragfähigkeit. Die Verwendung der Staatsausgaben ist dafür zentral: Wird mit den Schulden das Potenzialwachstum erhöht oder nicht? Auch eine stärkere Abhängigkeit zwischen Geld- und Fiskalpolitik droht. Stärker steigende Zinsen in den USA könnten zudem jene (Schwellen-)Länder treffen, die sich zur Überwindung der Pandemie und ihrer Folgen nicht in eigener Währung verschulden können. Die internationale Koordination von Geld- und Fiskalpolitik dürfte just zu einem Zeitpunkt schwieriger werden, da sie besonders benötigt wird. Das Comeback der Fiskalpolitik und ein mögliches makroökonomisches Paradigma der wirtschaftspolitischen Steuerung kommen somit nicht ohne Risiken.

\section{Literatur}

DeLong, J. B. (2021), What Are the New Inflation Hawks Thinking?, https://www.project-syndicate.org/commentary/american-rescueplan-inflation-critics-fear-monetary-policy-normalcy-by-j-bradforddelong-2021-03?barrier=accesspaylog.

King, M. (2021), Neue Zürcher Zeitung, 29. März, https://www.nzz.ch/ wirtschaft/mervyn-king-was-laeuft-ab-warum-geben-wir-so-vielgeld-aus-ld.1607909.

Krugman, P. (2021), https://www.bloomberg.com/news/articles/2021-03-18/ krugman-dismisses-1970s-style-inflation-risk-with-faith-in-fed.

Mason, J. W. (2021), The American Rescue Plan as Economic Theory, https:// jwmason.org/slackwire/the-american-rescue-plan-as-economic-theory/. OECD (2020), OECD Economic Outlook, 108, Dezember.

Summers, L. H. (2021), https://www.bnnbloomberg.ca/summers-says-us-facing-worst-macroeconomic-policy-in-40-years-1.1579791.

Summers, L. H. (2015), Demand Side Secular Stagnation, American Economic Review, 105(5), 60-65. 